\title{
A forehead hematoma as the initial clinical sign of lung cancer
}

\author{
Jonghyun Park, \\ Shin Hyuk Kang, \\ Woo Seob Kim, \\ Han Koo Kim, \\ Tae Hui Bae \\ Department of Plastic and \\ Reconstructive Surgery, Chung-Ang \\ University Hospital, Chung-Ang \\ University College of Medicine, Seoul, \\ Korea
}

\begin{abstract}
Primary lung cancer commonly metastasizes to the brain, bones, liver, and adrenal glands. In some cases, bone metastasis serves as the first presenting sign of lung cancer with bone pain and headache, but it is not common. The incidence of skull metastasis in lung squamous cell carcinoma (SCC) is low, and there have been only a few cases of skull metastases serving as the first sign of malignancy with skull mass and epidural bleeding; however, no similar cases have been reported regarding that of hematoma. We report a case of an 84-year-old man who first presented with a simple forehead hematoma and was eventually diagnosed with SCC of the lung.
\end{abstract}

Keywords: Hematoma / Lung neoplasm / Squamous cell carcinoma

\section{INTRODUCTION}

Lung cancer is one of the most common cancers with a high mortality rate, and it frequently metastasizes to the liver, adrenal glands, bone, and brain. Lung cancer is associated with bone metastases in approximately $36 \%$ of cancer patients [1,2]. Bone metastasis alone does not usually result in clinical presentation or diagnosis of the primary cancer, particularly when metastasis occurs in the skull. Only a few cases of skull metastases presenting as the first sign of malignancy have been reported [3-5]. Here, we described the case of a lung squamous cell carcinoma (SCC) in an 84-year-old man who first presented with a forehead hematoma caused by skull metastasis.

\section{CASE REPORT}

An 84-year-old man presented with a forehead hematoma that had occurred a month previously. The patient had no remarkable medical history except for a 30 pack-year smoking history

Correspondence: Woo Seob Kim

Department of Plastic and Reconstructive Surgery, Chung-Ang University

Hospital, 102 Heukseok-ro, Dongjak-gu, Seoul 06973, Korea

E-mail: kimws@cau.ac.kr

Received April 8, 2020 / Revised May 3, 2020 / Accepted June 19, 2020 and intermittent cough and sputum. He had suffered a minor contusion to his forehead a month ago that initially caused swelling, which then increased in size over the subsequent weeks. One week before he came to our hospital, he visited a local clinic where he received an incision and drainage procedure. However, the swelling recurred within days and continued to increase in size. He was referred to our hospital for further evaluation, where a physical examination revealed a hard and nontender solitary mass measuring $6.5 \times 6.0 \mathrm{~cm}$ with an unhealed incision site on the right forehead area (Fig. 1). He denied having skin lesions on his forehead prior to the trauma. Partial excision and biopsy of the lesion were performed under local anesthesia. The biopsy revealed moderately differentiated SCC with desmoplastic stromal reaction (Fig. 2).

The patient was hospitalized for further evaluation and treatment. Computed tomography (CT) scan showed a skull mass with destruction of the frontal bone and extension into the extracranial soft tissues (Fig. 3). A chest X-ray showed extensive opacity on the right lung, whereas a chest CT scan revealed a mass measuring $6.6 \times 7.4 \mathrm{~cm}$ in the right lower lobe with loculated pleural effusion (Fig. 4). The chest radiograph suggested lung carcinoma as the primary lesion. Further lung cancer evaluation was planned, but the patient rejected it, wanting only 

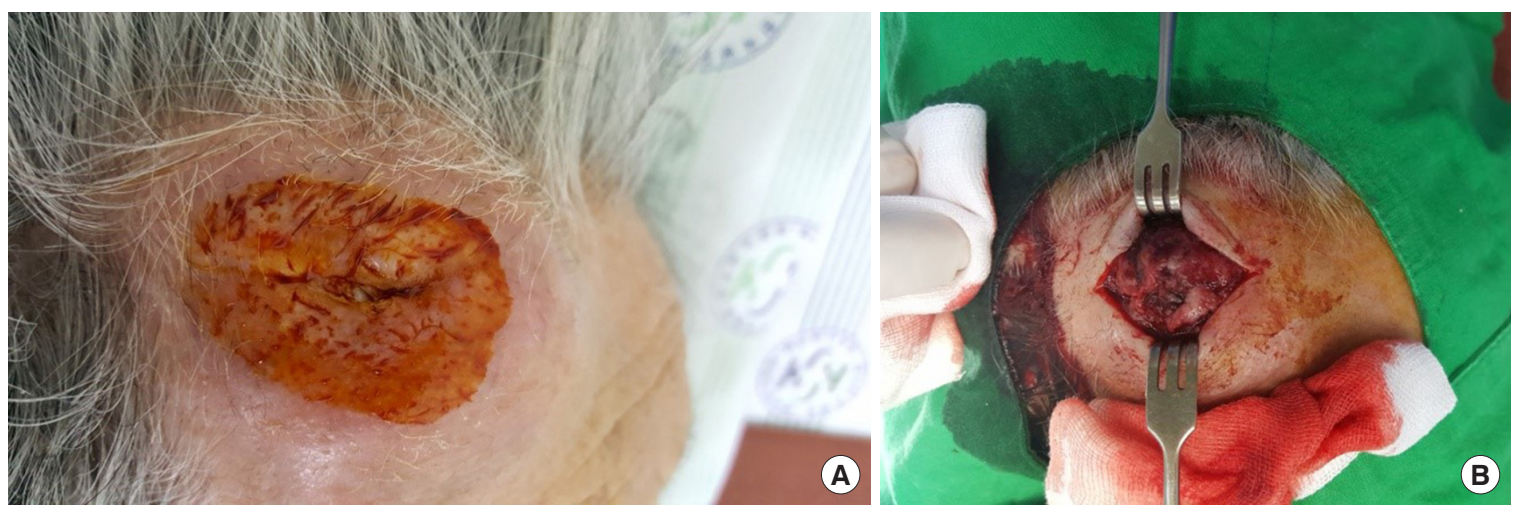

Fig. 1. An 84 -year-old man with a hard, non-tender, solitary mass measuring $6.5 \times 6.0 \mathrm{~cm}$ with an unhealed incision site. (A) Preoperative photograph. (B) Intraoperative photograph.
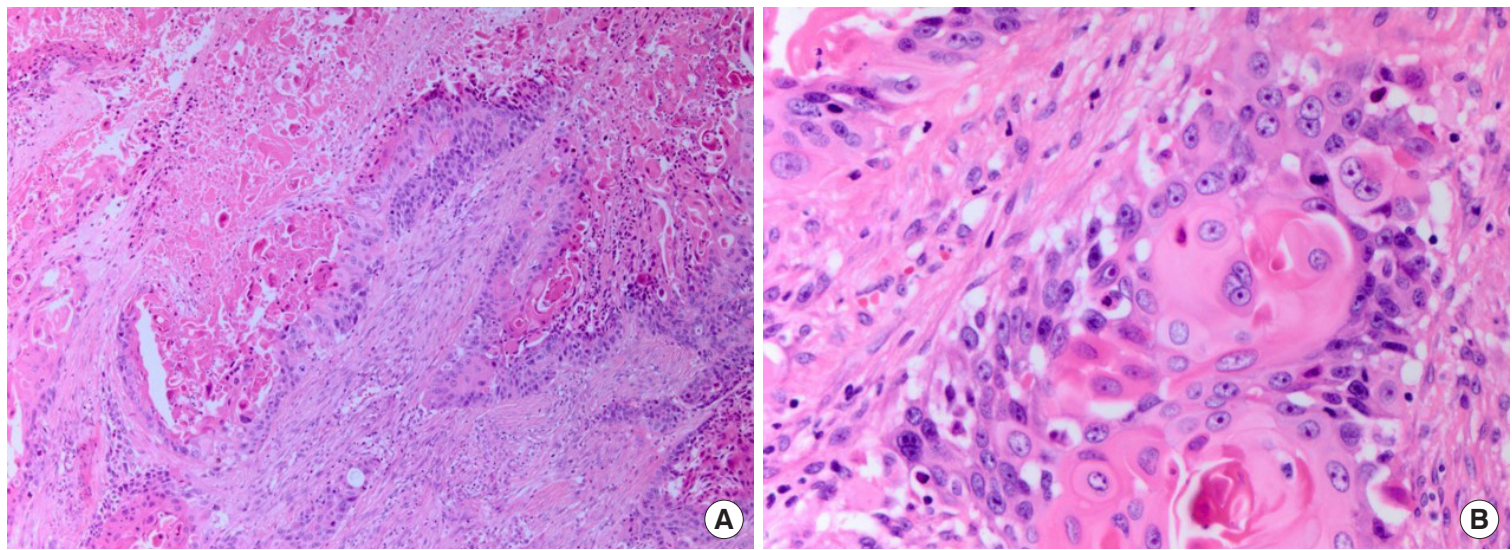

Fig. 2. Photomicrographs showing moderately differentiated squamous cell carcinoma with desmoplastic stromal reaction. (A) H\&E, $\times 100$; (B) $\mathrm{H} \& \mathrm{E}, \times 400$.

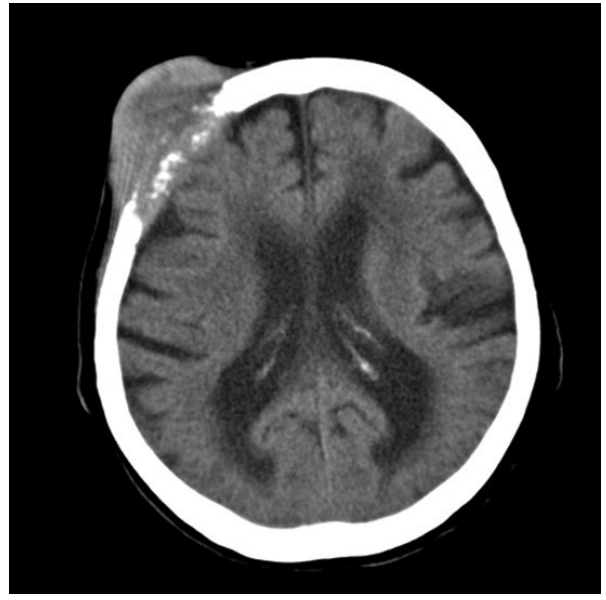

Fig. 3. Brain computed tomography scan showing a skull mass with frontal bone destruction and extension into the extracranial soft tissues.

conservative management. Consequently, simple debulking of the forehead mass was performed. Postoperative wound management was done for 3 weeks, but complete healing did not

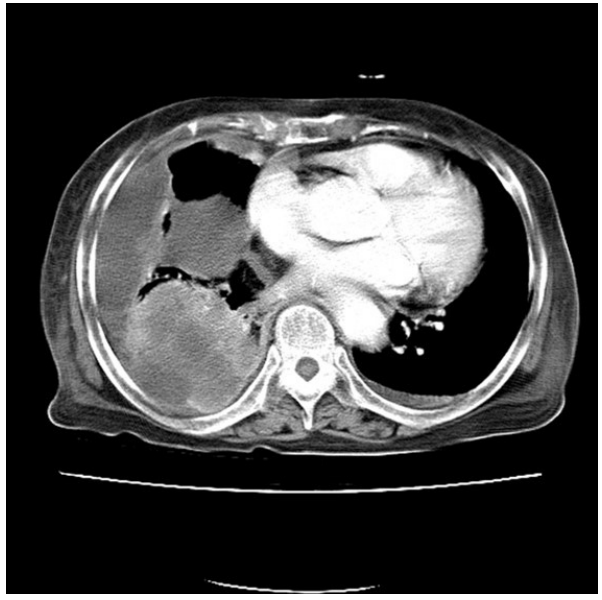

Fig. 4. Chest computed tomography scan showing a $6.6 \times 7.4 \mathrm{~cm}$ mass in the right lower lobe of the lung with loculated pleural effusion.

occur and upon the patient's request, he was transferred to a local clinic for subsequent wound management. 


\section{DISCUSSION}

Bone metastasis frequently occurs during the natural course of lung cancer, due partly to the advanced stage at which most lung cancers are diagnosed [4]. Skeletal metastases in the limbs and spine rarely serve as the first malignancy signs with bone pain. With only $3 \%$ of skeletal metastases being skull metastases, it is even more rare for skull metastases to serve as the first sign of lung cancer $[5,6]$. To best of our knowledge, this is the first study to report a forehead hematoma as the initial clinical sign of lung cancer.

In this case, a trauma history was misleading during diagnosis. Furthermore, verrucous lesions were only found under the subcutaneous layer, which delayed further evaluation. The actual pathogenesis of the forehead lesion is unknown. With given information of patient having no known history of skin lesions prior to the trauma, radiologic studies showing a skull mass with bony destruction and a large lung mass, and the biopsy revealing SCC with desmoplastic stromal reaction, which is associated with metastatic cancer, it is most likely that the lung cancer metastasis occurred beforehand and the trauma caused hematoma on the metastatic site [7]. Meanwhile, Fukushima et al. [8] had proposed another possibility with a case of a skull fracture triggering hepatocellular carcinoma metastasis to the fractured site, suggesting that the vascular endothelial and basic fibrous growth factors, involved in the bone healing process, are also involved in metastatic formation and may have induced metastasis. Although more study needs to be done, one possible pathogenesis could be the trauma triggering metastasis.

The treatment of lung cancer depends upon the tumor stage, histology, molecular characteristics, and patient's overall medical condition. Although no further assessment and treatment were done in the current case, with provided information on the size of the lung tumor greater than $7 \mathrm{~cm}$, no assessment of lymph node and one extrathoracic distant metastasis, the TNM staging for the disease was T4NxM1, stage IV. Patients with stage I, II, or III SCC lung cancer are generally treated with surgery, chemotherapy, radiation therapy, or a combined-modality approach, but palliative systemic therapy is the primary approach for patients with stage IV disease $[9,10]$.

Lung cancer is a molecularly heterogeneous disease and understanding its biology is crucial for the development of effective therapies [11]. Whenever possible, tumor tissue should be assessed for the expression of programmed cell death-ligand (PD-L1) and the presence of a somatic driver mutation of epidermal growth factor receptor (EGFR), anaplastic lymphoma kinase (ALK), c-ROS oncogene 1 (ROS1), and B-Raf (BRAF), which confer sensitivity to a specific inhibitor prior to the therapy choice. In advanced SCC lung cancer patients with tumor PD-L1 expression of $\leq 50 \%$, chemotherapy combined with immunotherapy is typically chosen, such as combination of pembrolizumab, carboplatin and either paclitaxel or nabpaclitaxel, with pembrolizumab maintenance after the initial four to six cycles is recommended. For SCC lung cancer patients with PDL1 expression $\geq 50 \%$, pembrolizumab monotherapy is recommended [9].

In conclusion, trauma could serve as both misleading and diagnostic factor for the diagnosis of lung cancer. Trauma-induced skin lesions require careful examination to detect fractures and additional pathologies. As demonstrated, a simple hematoma could be the first malignancy sign. Trauma history could mislead, but careful and thorough examination of the patient could turn misleading factor into a diagnostic factor of malignancy. While more studies are required to determine the actual pathogenesis of skull metastasis of lung cancer in this case, achieving accurate clinical diagnosis and avoiding misdiagnosis is the highest priority.

\section{NOTES}

\section{Conflict of interest}

No potential conflict of interest relevant to this article was reported.

\section{Ethical approval}

The study was approved by the Institutional Review Board of Chung-Ang University (IRB No. 2004-008-19310) and performed in accordance with the principles of the Declaration of Helsinki. Written informed consent was obtained.

\section{Patient consent}

The patient provided written informed consent for the publication and the use of his images.

\section{ORCID}

Jonghyun Park https://orcid.org/0000-0001-9910-6945

Shin Hyuk Kang https://orcid.org/0000-0001-9868-2019

Woo Seob Kim https://orcid.org/0000-0002-4104-3926

Han Koo Kim https://orcid.org/0000-0002-2849-3973

Tae Hui Bae https://orcid.org/0000-0002-0342-1439

\section{REFERENCES}

1. Coleman RE. Clinical features of metastatic bone disease and risk of skeletal morbidity. Clin Cancer Res 2006;12(20 Pt 2): 
6243s-6249s.

2. Sugiura H, Yamada K, Sugiura T, Hida T, Mitsudomi T. Predictors of survival in patients with bone metastasis of lung cancer. Clin Orthop Relat Res 2008;466:729-36.

3. Hayashi K, Matsuo T, Kurihara M, Daikoku M, Kitange G, Shibata S. Skull metastasis of hepatocellular carcinoma associated with acute epidural hematoma: a case report. Surg Neurol 2000;53: 379-82.

4. Santini D, Barni S, Intagliata S, Falcone A, Ferrau F, Galetta D, et al. Natural history of non-small-cell lung cancer with bone metastases. Sci Rep 2015;5:18670.

5. Turner RC, Lucke-Wold BP, Hwang R, Underwood BD. Lung cancer metastasis presenting as a solitary skull mass. J Surg Case Rep 2016;2016:rjw116.

6. Garcia Carretero R, Sanchez-Redondo J, Barrio-Alonso MJ, Lopez-Marti MP. Lung carcinoma presenting as a solitary, painless frontal bone lump. BMJ Case Rep 2015;2015:bcr2015212038.

7. Lonsdorf AS, Hadaschik EN. Squamous cell carcinoma and keratoacanthoma. In: Kang S, Amagai M, Bruckner AL, Enk
AH, Margolis DJ, McMichael AJ, et al, editors. Fitzpatrick's dermatology. 9th ed. New York: McGraw-Hill Education; 2019. p. 1901-19.

8. Fukushima M, Katagiri A, Mori T, Watanabe T, Katayama Y. Case of skull metastasis from hepatocellular carcinoma at the site of skull fracture. No Shinkei Geka 2010;38:371-7.

9. Lilenbaum RC. Systemic chemotherapy for advanced nonsmall cell lung cancer [Internet]. Waltham, MA: UpToDate; c2020 [cited 2020 Apr 29]. Available from: https://www.uptodate.com/contents/systemic-chemotherapy-for-advancednon-small-cell-lung-cancer.

10. Goldstraw P, Chansky K, Crowley J, Rami-Porta R, Asamura H, Eberhardt WE, et al. The IASLC Lung Cancer Staging Project: Proposals for Revision of the TNM Stage Groupings in the Forthcoming (Eighth) Edition of the TNM Classification for Lung Cancer. J Thorac Oncol 2016;11:39-51.

11. Herbst RS, Morgensztern D, Boshoff C. The biology and management of non-small cell lung cancer. Nature 2018;553:44654. 